\title{
TP53I11 suppresses epithelial-mesenchymal transition and metastasis of breast cancer cells
}

\author{
Tongqian Xiao ${ }^{1,2}$, Zhongjuan Xu ${ }^{1}$, Hai Zhang ${ }^{1}$, Junsa Geng ${ }^{1,4}$, Yong Qiao ${ }^{1}$, Yu Liang ${ }^{1,3}$, Yanzhen Yu ${ }^{1,4}$, Qun Dong ${ }^{5, *} \mathcal{E}^{*}$ \\ Guangli Suo ${ }^{1, *}$ \\ ${ }^{1}$ CAS Key Laboratory of Nano-Bio Interface, Suzhou Institute of Nano-Tech and Nano-Bionics, Chinese Academy of Sciences, Jiangsu \\ 215123, ${ }^{2}$ University of Chinese Academy of Sciences, Beijing 100049, ${ }^{3}$ School of Life Sciences, Shanghai University, Shanghai 200444, \\ ${ }^{4}$ University of Science and Technology of China, Anhui 230026, ${ }^{5}$ Taikang National Pathology Center, TaikangXianlin Drum Tower \\ Hospital, Jiangsu 210046, China
}

\begin{abstract}
Epithelial-mesenchymal transition (EMT) is widely-considered to be a modulating factor of anoikis and cancer metastasis. We found that, in MDA-MB-231 cells, TP53I11 (tumor protein P53 inducible protein 11) suppressed EMT and migration in vitro, and inhibited metastasis in vivo. Our findings showed that hypoxic treatment upregulated the expression of HIF1 $\alpha$, but reduced TP53I11 protein levels and TP53I11 overexpression reduced HIF1 $\alpha$ expression under normal culture and hypoxicconditions, and in xenografts of MDA-MB-231 cells. Considering HIF1 $\alpha$ is a master regulator of the hypoxic response and that hypoxia is a crucial trigger of cancer metastasis, our study suggests that TP53I11 may suppress EMT and metastasis by reducing HIF1 $\alpha$ protein levels in breast cancer cells. [BMB Reports 2019; 52(6): 379-384]
\end{abstract}

\section{INTRODUCTION}

Metastasis, an important characteristic of malignant tumors, is one of the most life-threatening pathological events (1), and is the end-product of a multi-step cell-biological process (2). Epithelial-mesenchymal transition (EMT), which has been well-characterized in embryonic development, is widelyconsidered to be a modulating factor for anoikis and cancer metastasis (3-7). Many studies have suggested that the loss of epithelial features and the acquisition of mesenchymal properties are the major characteristics necessary for the progression of breast cancer (8).

${ }^{*}$ Corresponding authors. Guangli Suo, Tel: +86-512-62872823; Fax: +86-512-62872569; E-mail: glsuo2013@sinano.ac.cn; Qun Dong, Tel: +86-25-85358120; Fax: +86-25-6875-9803; E-mail: td000777 @gmail.com

https://doi.org/10.5483/BMBRep.2019.52.6.173

Received 24 July 2018, Revised 15 August 2018, Accepted 2 October 2018

Keywords: EMT, HIF1 $\alpha$, Hypoxia, Metastasis, TP53I11
Hypoxia is a hallmark of solid tumors, and can activate hypoxia inducible factors (HIFs). As a group of DNA binding proteins, HIFs participate in the regulation of angiogenesis, glycolysis, metabolic adaptation, erythropoiesis, cell survival, EMT, and metastasis $(9,10)$. HIF1 $\alpha$ was reported to induce EMT and metastasis by regulating EMT regulators, including TWIST, vimentin, Slug, and E-cadherin (11).

TP53I11 (tumor protein P53-inducible protein 11) was first reported as one of the early transcriptional targets of TP53 (12). So far, the studies on TP53I11 were mostly focused on the positive effect of TP53111 on apoptosis as a tumor suppressor in cancer cells $(13,14)$. However, the underlying mechanisms of TP53I11 are still unclear. We found that TP53I11 suppressed EMT and the metastasis of breast cancer cells. Hypoxic stress decreased endogenous TP53I11 protein levels in MDA-MB-231 cells. The overexpression of TP53I11 suppressed HIF1 $\alpha$ protein levels in cells under both normal and hypoxic culture conditions. The results suggest that TP53I11 may affect EMT and metastasis through regulation of HIF1 $\alpha$.

\section{RESULTS}

TP53I11 inhibits mesenchymal traits

MCF10A is an immortalized human non-tumorigenic mammary epithelial cell line and is widely-used for in vitro studies of normal breast cell function and transformation. MDA-MB-231 is a highly-metastatic breast cancer cell line with typical mesenchymal traits. Our previous study showed that TP53I11 promoted anoikis in MCF10A and MDA-MB-231 cells (15). Anoikis is regarded as a hallmark of oncogenic EMT. To determine if TP53I11 was associated with EMT, we constructed a TP53I11-overexpressing breast cancer MDA-MB231 cell line (M231-P11-OE) and its vector control cells (M231-V-OE) (Fig. S1A). The effect of TP53I11 on the expression of EMT markers was examined using MDA-MB-231 cells (Fig. 1A and B). We found that TP53I11 overexpression reduced the expression of $\mathrm{CDH} 2$ (N-cadherin), VIM (vimentin), SNAI2 (Slug), and SNAI1 (Snail), and enhanced the

ISSN: 1976-670X (electronic edition)

Copyright (C) 2019 by the The Korean Society for Biochemistry and Molecular Biology

(c) This is an open-access article distributed under the terms of the Creative Commons Attribution Non-Commercial License (http://creativecommons.org/licenses/by-nc/4.0) which permits unrestricted non-commercial use, distribution, and reproduction in any medium, provided the original work is properly cited. 
A

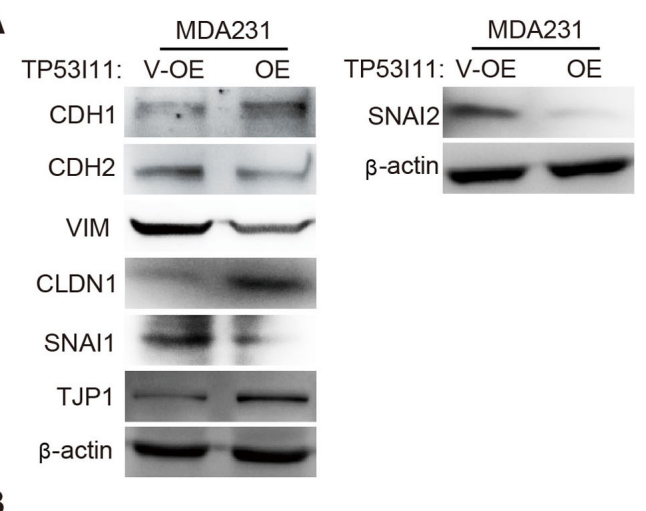

B

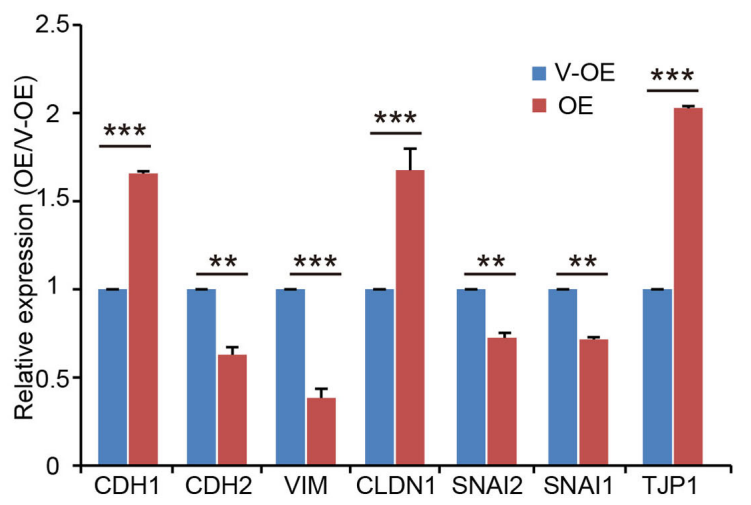

Fig. 1. TP53I11 reduces the protein levels of mesenchymal markers in MDA-MB-231. (A) Immunoblotting assay of EMT markers in MDA-MB-231 derived cells. (B) Quantification of the relative protein expression (normalized to $\beta$-actin) in immunoblots. V-OE, vector control for overexpression; OE, overexpression; MDA231, MDA-MB-231 cell line. $* * P<0.01, * * * P<0.005 . P$ values were determined using a two-tailed student's t-test. Error bars represent the S.D.s from the average of at least three biological replicates.

expression of $\mathrm{CDH} 1$ (E-cadherin), CLDN1 (claudin-1), and TJP1 (zonaoccludens 1, ZO-1) (Fig. 1A and B). These results showed the negative effect of TP53I11 on mesenchymal transition.

\section{TP53I11 inhibits cell migration}

Considering mesenchymal transition is required for cell migration, we therefore investigated if TP53I11 also affected on the migration and invasion of MDA-MB-231 cells (Fig. 2). The results showed that overexpression of TP53I11 suppressed, and loss of TP53I11 promoted, wound closure, cell migration, and invasion in MDA-MB-231 cells (Fig. 2).

\section{TP53I11 inhibits tumor metastasis in vivo}

We transplanted M231-V-OE and M231-P11-OE cells into $\mathrm{BALB} / \mathrm{c}$ female nude mice by orthotopic injection into the mammary fat pads or by tail-vein injection. According to the immunohistochemical (IHC) staining results of tumor sections in the orthotopic injections, we found that TP53I11 overexpression reduced the expression of mesenchymal markers hVIM and $\mathrm{CDH} 2$ (Fig. 3A). Three distinct layers in primary tumors were detected by hematoxylin and eosin (H\&E) staining. The necrotic tumor center (n) was surrounded by a healthy, viable rim (v), and the outermost layer was dermis (Fig. 3B and D). Compared with the control M231-V-OE tumors, the M231-P11-OE tumors generally demonstrated a smaller viable tumor rim (Fig. 3B and D). We found that TP53I11 overexpression substantially suppressed local invasion (Fig. 3B and C) and significantly reduced the metastatic burden in mice lungs (Fig. $3 \mathrm{E}$ and $\mathrm{F}$ ). The colonization of breast cancer cells into the lungs was also measured by $\mathrm{H} \& \mathrm{E}$ and $\mathrm{IHC}$ for human VIM in mice with tail-vein injections (Fig. 3G and H). We found that TP53I11 overexpression significantly reduced the number and the size of tumor colonies in the lungs (Fig. 3l). These in vivo results confirmed our in vitro findings that TP53I11 suppressed EMT, cell migration, and invasion.

\section{Hypoxia treatment decreases TP53I11 protein levels and TP53I11 reduces HIF1 $\alpha$ protein levels} In our study, under hypoxic conditions, HIF1 $\alpha$ expression was upregulated, as previously reported (Fig. 4A and B) (16), but, the expression level of TP53I11 in MDA-MB-231 cells was markedly decreased (Fig. 4A and C). A few studies have reported that TP53111 functioned as a tumor suppressor in some cancer cells $(13,14)$, and HIF1 $\alpha$ acted a crucial role in EMT and metastasis $(11,17)$. Here, we found that overexpression of TP53I11 decreased, and loss of TP53I11 increased, HIF1 $\alpha$ expression under normal culture and hypoxic conditions (Fig. 4D-I). IHC staining of tumor sections showed that overexpression of TP53I11 reduced the HIF1 $\alpha$ expression in MDA-MB-231 xenografts (Fig. 4J). The results in Fig. 4 indicate that TP53I11 may suppress the EMT and metastasis by inhibiting HIF1 $\alpha$ expression in MDA-MB-231 cells.

\section{DISCUSSION}

Our previous research demonstrated that TP53I11 promoted anoikis in MCF10A and MDA-MB-231 cells (15). The fact that anoikis has been widely-considered to be a hallmark of oncogenic EMT motivated us to investigate if, and how TP53I11 affected EMT and cell migration in MDA-MB-231 cells. In this study, we reported that TP53I11 suppressed EMT and cell migration in MDA-MB-231 cells, and inhibited metastasis to mice lungs in vivo. This work supports other findings in which TP53I11 was regarded to be a tumor suppressor gene $(13,18)$.

As a key mediator of cellular adaptation to hypoxia, HIF1 $\alpha$ enhances cell EMT and tumor metastasis $(17,19)$. Under hypoxic conditions, HIF1 $\alpha$ was stabilized and translocated 
A

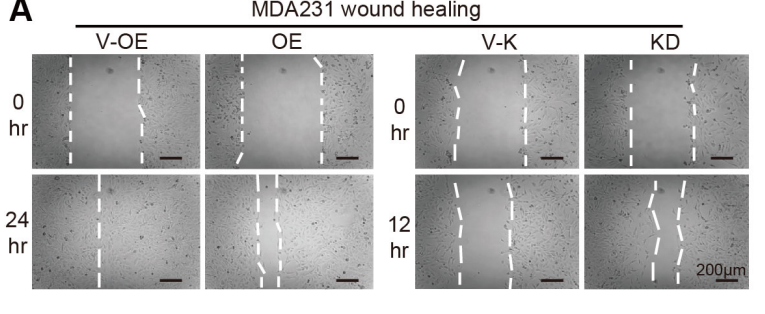

C

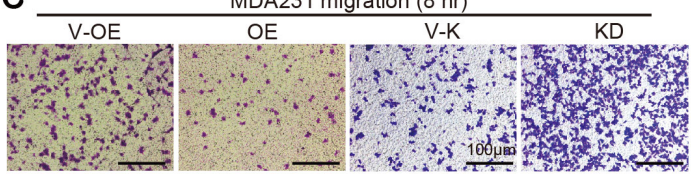

E MDA231 invasion (24 hr)

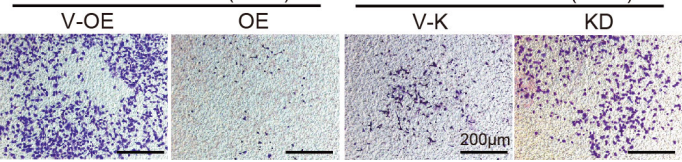

B

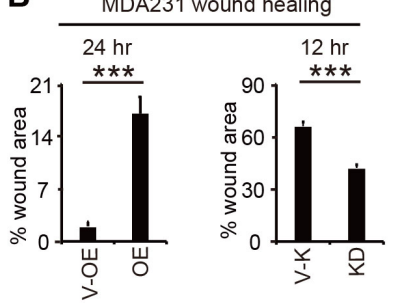

Fig. 2. TP53I11 suppresses migration and invasion in MDA-MB-231. Representative images of wound healing assays (A), transwell migration assays (C), and invasion assays (E) of MDAMB-231 derived cells. The images were captured at the indicated times of cell culture. The percentages of wound areas were calculated and shown in (B). The number of migrated cells and invaded cells in each visual field were counted and shown in (D) and (F), respectively. $\mathrm{V}-\mathrm{OE}$, vector control for overexpression; $\mathrm{OE}$, overexpression; V-K, vector control for knockdown or knockout; KD, knockdown; **P $<0.01, * * * P<0.005$ $P$ values were determined using a two-tailed student's t-test. Error bars represent the S.D.s from the average value of at least three biological replicates.

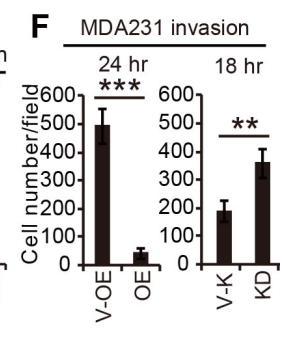

into the nucleus, which resulted in formation of an active HIF1 $\alpha$ transcription factor (20). TP53I11 has been reported to be a target gene of Mir-210 (21), and Mir-210 was directly regulated by HIF1 $\alpha$ (22). This suggests that HIF1 $\alpha$ may regulate TP53I11 expression by regulation of Mir-210. We found that the expression level of HIF1 $\alpha$ was upregulated, but TP53I11 expression was reduced by hypoxic treatment. Moreover, HIF1 $\alpha$ expression was upregulated by the loss of TP53111 and downregulated by the overexpression of TP53I11. We also found that TP53I11 exerted no effect on HIF1 $\alpha$ transcription (data are not shown). These findings suggest that the TP53I11 may have regulated HIF1 $\alpha$ protein accumulation in MDA-MB-231 cells, which then affected EMT and cancer metastasis. Of course, whether HIF1 $\alpha$ regulates TP53111 expression by regulation of Mir-210, and how TP53I11 affects HIF1 $\alpha$ accumulation needs more research in the future. Overall, for the first time, we reported the effect of TP53I11 on EMT and breast cancer metastasis and provided new insight into the mechanism of TP53I11 as a tumor suppressor.

\section{MATERIALS AND METHODS}

\section{Cell culture}

MDA-MB-231 (ATCC, Manassas, VA, USA) cells were grown in complete Dulbecco's Modified Eagle Medium (DMEM) (Gibco) supplemented with 10\% fetal bovine serum and 1\% penicillin/streptomycin under humidified conditions in 95\% air and $5 \% \mathrm{CO}_{2}$ at $37^{\circ} \mathrm{C}$ (3). For hypoxic treatment, MDAMB-231 were cultured for 12 hours in $1 \%$ oxygen, $5 \%$ carbon dioxide, and $94 \%$ nitrogenin a hypoxia chamber as previously described (15).
Plasmids construction, viral production, and transduction The human TP53I11 CDNA was cloned from MDA-MB-231 cells by RT-PCR with primers (Table S1) and inserted into a pQCXIH retrovector (Clontech Laboratories, Mountain View, CA). TP53I11 and a control vector retrovirus were produced and used to infect cells for $48 \mathrm{hr}$. After infecting, the cells were selected with $100 \mu \mathrm{g} / \mathrm{ml}$ hygromycin B. The shRNA for TP53I11 knockdown (Table S1) was cloned into a pLKO.1 vector. Cells were infected with TP53I11-shRNA lentivirus for $48 \mathrm{hr}$ and selected with $1 \mu \mathrm{g} / \mathrm{ml}$ puromycin. Overexpression or knockdown of genes was evaluated by qRT-PCR using the primers shown in Table S1 and by immunoblotting.

Quantitative real-time polymerase chain reaction (qRT-PCR) The qRT-PCR was performed according to our previous study (3). The qRT-PCR primers for TP53I11 (F: 5'-GGCTCAGGG TCTGGCAGTT-3'; R: 5'-CCATCAAAGACCGCATCATAGA-3') and GAPDH (F: 5'-ACCCACTCCTCCACCTTTGA-3'; R: 5'-TGTTGCTGTAGCCAAATTCGTT-3') were designed using Primer Express software (version 2.0, Applied Biosystems).

\section{Immunoblotting}

Cell lysates were prepared in lysis buffer $(25 \mathrm{mM}$ Tris $\mathrm{pH} 7.4$, $150 \mathrm{mM} \mathrm{NaCl}, 1 \mathrm{mM}$ EDTA, $10 \%$ glycerol, $1 \%$ Nonidet P-40, $0.1 \%$ SDS, and $1 \%$ sodium deoxycholate) supplemented with $1 \times$ protease cocktail. The lysates were cleared by centrifugation at $21,000 \times \mathrm{g}$ for 15 minutes at $4^{\circ} \mathrm{C}$, separated on SDS-PAGE gels, and transferred to polyvinylidene difluoride (PVDF) membranes. The membranes were blocked with $5 \%$ non-fat milk for $1 \mathrm{hr}$ and incubated with primary antibodies overnight at $4^{\circ} \mathrm{C}$, followed by incubation with the corresponding HRP-conjugated secondary antibody. Finally, the membranes were visualized by chemiluminescence. The 
A

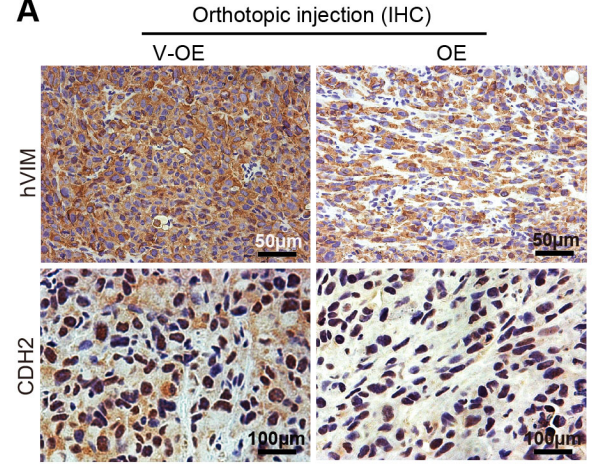

E

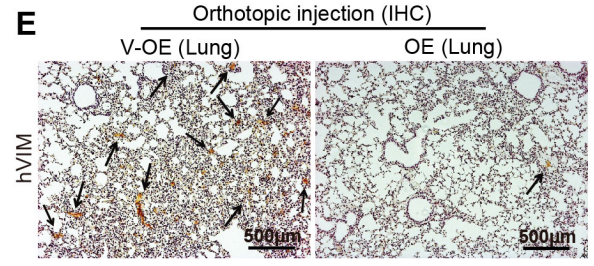

G

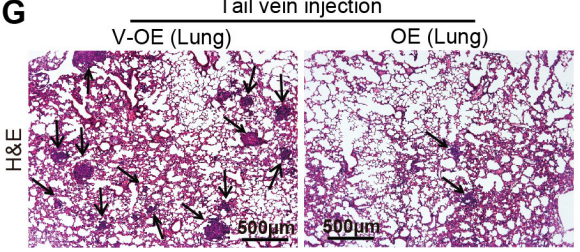

B

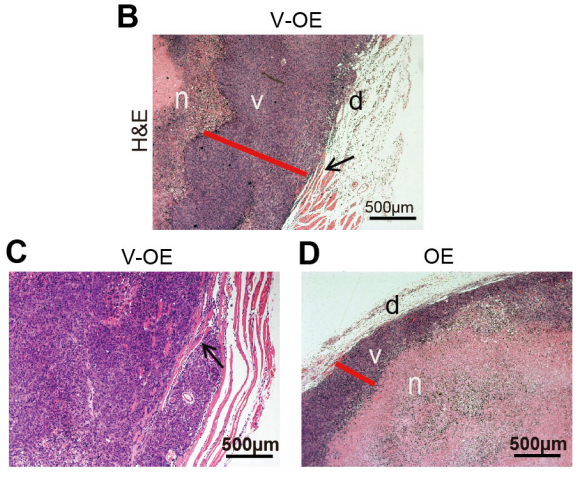

$\mathbf{F}$

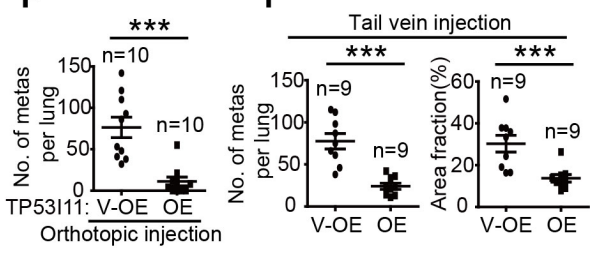

H

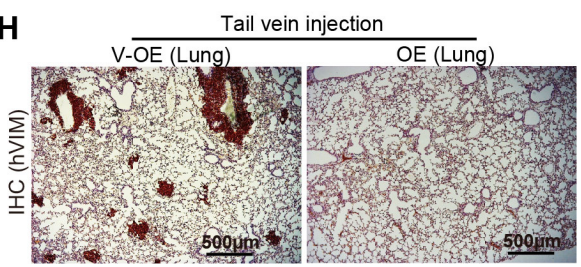

Fig. 3. TP53I11 overexpression reduces MDA-MB-231 cells metastasis in vivo. Orthotopic injection: 231-P11-OE (OE) and vector control 231-V-OE (V-OE) cells were injected into the mammary fat pads of immunodeficient mice. Mice were euthanized 48 days later. (A) The tumors were sectioned and stained for hVIM and CDH2, and with hematoxylin and eosin (H\&E). The necrotic center " $\mathrm{n}$ " is surrounded by a section of viable tumor rim " $v$ " with an outer dermal layer " $d$ " (B-D). TP53I11 overexpression reduced tumor invasion into nearby tissues. Black arrows point to the invasion locations. Red lines show the thickness of the viable tumor rim. Scale bar $=500 \mu \mathrm{m}$. (E) IHC staining for hVIM in lung tissue sections and (F) hVIM-positive nodules in the lungs of mice with orthotopic injections. Tail vein injection: 231-P11-OE (OE) and vector control 231-V-OE (V-OE) cells were injected into immunodeficient mice through the tail veins and the mice were sacrificed 35 days later. $(\mathrm{G}) \mathrm{H} \& \mathrm{E}$ and $(\mathrm{H}) \mathrm{IHC}(\mathrm{hVIM})$ staining of lung tissue sections. (I) The number and size of metastatic nodules. $\mathrm{V}-\mathrm{OE}$, vector control for overexpression; $\mathrm{OE}$, overexpression. ${ }^{* * *} \mathrm{P}<0.005$. $\mathrm{P}$ values were determined using a two-tailed student's t-test.Results are expressed as mean \pm SD from the indicated number of mice.

band intensities were analyzed with Image $J$ software and normalized to $\beta$-actin or GAPDH. Antibodies against the following proteins were purchased from Cell Signaling Technology (Beverly, MA, USA): GAPDH, $\beta$-actin, $N$-cadherin $(\mathrm{CDH} 2)$, E-cadherin $(\mathrm{CDH} 1)$, vimentin (VIM), Slug (SNAI2), Snail (SNAI1), claudin-1 (CLDN1), ZO-1 (TJP1), and TCF8/ZEB1 (ZEB1).The antibodies against $\mathrm{N}$-cadherin $(\mathrm{CDH} 2)$ and vimentin (VIM) used for IHC were purchased from ImmunoWay (Plano, TX, USA). The goat anti-rabbit or anti-mouse HRP-conjugated secondary antibodies were also purchased from Cell Signaling Technology (Beverly, MA, USA). The primary antibody against TP53I11 was generated by GL Biochem Ltd (Shanghai, China). Briefly, three mice were immunized three times with the YDAVFDGAQVTSKTP polypeptide. Serum was obtained from blood samples and the specificity of the antibody against TP53I11 was confirmed by immunoblotting using a FLAG antibody against TP53111-FLAG fusion protein.

\section{Wound healing, Transwell migration, and Matrigel invasion assays}

For wound healing, cells were seeded into 6-well plates at a density of $2 \times 10^{5}$ cells per well and cultured at $37^{\circ} \mathrm{C}$ in growth medium. Monolayers with $90 \%$ confluency were starved overnight and a single scratch wound was created using a $200 \mu \mathrm{l}$ micropipette tip. Wounds were visualized and images were captured by phase-contrast microscopy (Nikon Eclipse TS100) at the indicated hours post-wounding. Areas of the wounds were measured by ImageJ software and normalized to the initial wound area. The migration and 
A

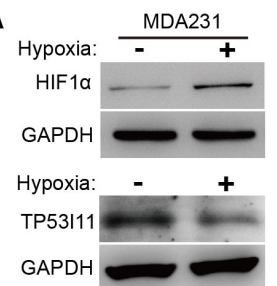

E

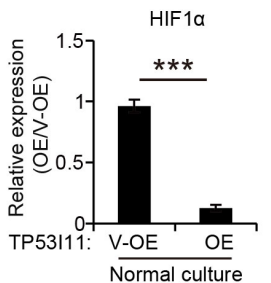

G

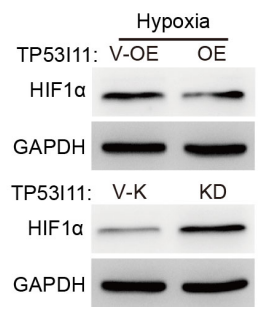

B

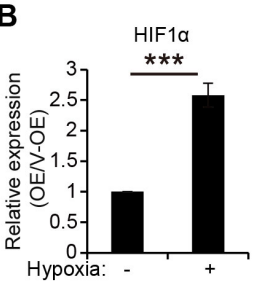

F
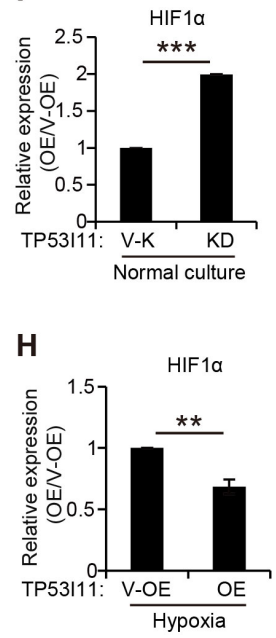

C

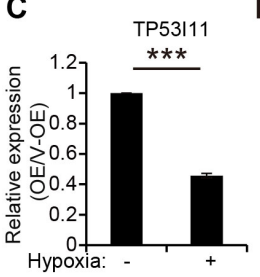

$\mathbf{J}$
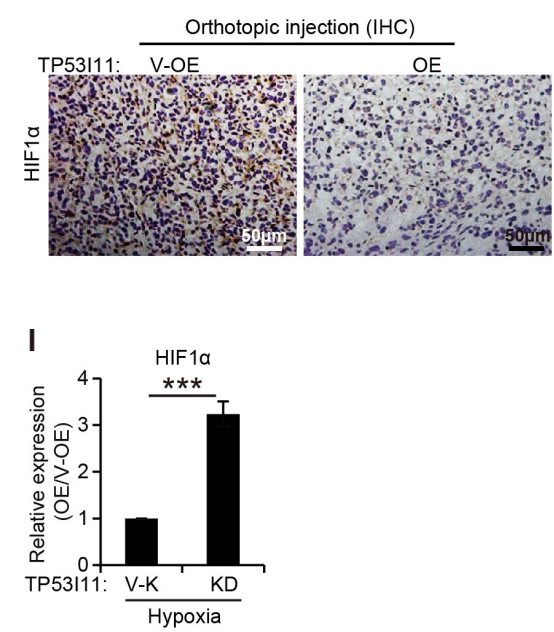

D

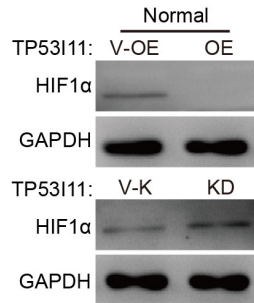

Fig. 4. TP53I11 reduces the HIF1 $\alpha$ protein level. (A) Immunoblotting assays showing decreased protein levels of TP53I11 and increased HIF1 $\alpha$ after hypoxic treatment. (B and $\mathrm{C}$ ) Quantification of the relative protein expression (normalized to GAPDH) in immunoblots. The HIF1 $\alpha$ protein levels were also examined in MDA-MB-231derived cells under normal culture $(\mathrm{D})$, hypoxic treatment $(\mathrm{G})$ and in vivo (J). (E, F, H, and I) Quantification of the relative protein expression (normalized to GAPDH) in immunoblots. V-OE, vector control for overexpression; $\mathrm{OE}$, overexpression; $\mathrm{V}-\mathrm{K}$, vector control forknockdown or knockout; KD, knockdown. ${ }^{* * P}<0.01$, $* * * \mathrm{P}<0.005$. $\mathrm{P}$ values were determined using a two-tailed student's t-test. Error bars represent the S.D.s from the average of at least three biological replicates. invasion assays were performed according to our previous study (3). For the migration assay, MDA-MB-231-derived cells were starved overnight, then plated in the top upper transwell chambers ( $8 \mu \mathrm{m}$ pore size; Corning) and cultured in growth medium. Growth medium $(500 \mu \mathrm{l})$ was added to each bottom lower chamber as an attractant for migration. At the indicated times, cells on the basal side of the membrane were fixed with $100 \%$ methanol and stained with $0.1 \%$ crystal violet solution. For the invasion assay, each upper chamber was first coated with $50 \mu \mathrm{l}$ basement membrane growth factor-reduced Matrigel, then seeded with cells and incubated for the indicated hours. The number of cells on the basolateral microporous membrane was determined as described above for the transwell migration assay.

\section{Metastasis assays}

The metastasis assays were conducted with our previous methods (3). For orthotopic inoculation, cells $\left(6 \times 10^{6}\right.$ in 60 $\mu$ ) were injected orthotopically into the mammary fat pad of 6-week-old female nude mice. All animals were sacrificed 48 days after the initial implantation. Primary tumors and lung tissues were collected and fixed in $4 \%$ paraformaldehyde for further analyses. For tail inoculation, $1 \times 10^{6}$ viable cells were injected into the lateral tail vein. All animals were sacrificed after 35 days of inoculation. Lung tissues were collected and fixed in $4 \%$ paraformaldehyde for further analyses. Paraformaldehyde-fixed tumors and lungs were paraffin- embedded and sectioned (5 $\mu \mathrm{m})$. Hematoxylin and eosin (H\&E) staining and immunohistochemical (IHC) assays using antibodies, such as anti- $\mathrm{N}$-cadherin $(\mathrm{CDH} 2)$ and anti-human vimentin (hVIM) were performed. The stained sections were observed and the images were acquired using a microscope (ZEISS AXIO microscope, Axio Scope A1). The number of metastatic nodules in each lung was calculated. ImageJ Processing and Analysis software was used to quantify areas of abnormal tissue, which were defined as tissue that appeared as areas of solid mass compared to normal sparsely-looking tissue. The percentage of abnormal lung tissue was calculated using the following formula: ((area of abnormal tissue / total tissue area) $\times 100$ ). Three sections were used for each animal.

\section{Statistical analysis}

All data are expressed as mean \pm the standard error of the mean from at least three independent experiments. The mean and S.D. were calculated using Microsoft Excel or GraphPad Prism 5 software. Statistical significance was determined by one-way ANOVA or unpaired two-tailed student's t-test and P $<0.05$ was considered statistically significant.

\section{ACKNOWLEDGEMENTS}

We thank Jianwu Dai, Qiangbin Wang, Zhijun Zhang, Renjun Pei, and Guosheng Cheng for insightful comments on the study, Xiang $\mathrm{He}$ for her excellent technical assistance. 
Nano-Bio-Chem Centre in Suzhou Institute of Nano-Tech and Nano-Bionics (SINANO) is acknowledged for professional assistance with cell imaging and FACS assays. This work was supported by the National Natural Science Foundation of China (Grant No. 31471307); and the Ministry of Science and Technology (MOST) (Grant No. 2017YFA0104301 and 2014CB965003). G. Suo was also supported by the Hundred Talent Program of the Chinese Academy of Sciences.

\section{CONFLICTS OF INTEREST}

The authors have no conflicting interests.

\section{REFERENCES}

1. Yilmaz M and Christofori G (2009) EMT, the cytoskeleton, and cancer cell invasion. Cancer Metastasis Rev 28, 15-33

2. Valastyan S and Weinberg RA (2011) Tumor metastasis: molecular insights and evolving paradigms. Cell 147, 275-292

3. Zhou Y, Xu Z, Quan D et al (2018) Nuclear respiratory factor 1 promotes spheroid survival and mesenchymal transition in mammary epithelial cells. Oncogene 37, 6152-6165

4. Au SH, Storey BD, Moore JC et al (2016) Clusters of circulating tumor cells traverse capillary-sized vessels. Proc Natl Acad Sci U S A 113, 4947-4952

5. Grimshaw MJ, Cooper L, Papazisis K et al (2008) Mammosphere culture of metastatic breast cancer cells enriches for tumorigenic breast cancer cells. Breast Cancer Res 10, R52

6. Dontu G, Abdallah WM, Foley JM et al (2003) In vitro propagation and transcriptional profiling of human mammary stem/progenitor cells. Genes Dev 17, 12531270

7. Al-Hajj M, Wicha MS, Benito-Hernandez A, Morrison SJ and Clarke MF (2003) Prospective identification of tumorigenic breast cancer cells. Proc Natl Acad Sci U S A 100, 3983-3988

8. Kokkinos MI, Wafai R, Meng KW et al (2007) Vimentin and Epithelial-Mesenchymal Transition in Human Breast Cancer - Observations in vitro and in vivo. Cells Tissues Organs 185, 191-203

9. Semenza GL (2012) Hypoxia-inducible factors in physiology and medicine. Cell 148, 399-408
10. Moon Y, Park B and Park H (2016) Hypoxic repression of CYP7A1 through a HIF-1alpha- and SHP-independent mechanism. BMB Rep 49, 173-178

11. Koh Y, Byeon H, Kim W et al (2012) 458 Hypoxiainducible Factor 1a (HIF-1a) Induces Epithelialmesenchymal Transition (EMT) in Thyroid Cancer Cell Lines. Eur J Cancer 48, 142-142

12. Polyak K, Xia Y, Zweier JL, Kinzler KW and Vogelstein B (1997) A model for p53-induced apoptosis. Nature 389, 300-305

13. Ramachandran C, Rodriguez S, Ramachandran $R$ et al (2005) Expression profiles of apoptotic genes induced by curcumin in human breast cancer and mammary epithelial cell lines. Anticancer Res 25, 3293-3302

14. Wu Y, Liu XM, Wang XJ et al (2009) PIG11 is involved in hepatocellular carcinogenesis and its over-expression promotes Hepg2 cell apoptosis. Pathol Oncol Res 15, $411-416$

15. Xiao T, Xu Z, Zhou Y et al (2019) Loss of TP53I11 Enhances the Extracellular Matrix-independent Survival by Promoting Activation of AMPK. IUBMB Life 71, 183-191

16. Ghoshal P, Teng Y, Lesoon LA and Cowell JK (2012) HIF1A induces expression of the WASF3 metastasisassociated gene under hypoxic conditions. Int J Cancer 131, E905-915

17. El-Naggar AM, Veinotte CJ, Cheng $\mathrm{H}$ et al (2015) Translational Activation of HIF1alpha by YB-1 Promotes Sarcoma Metastasis. Cancer Cell 27, 682-697

18. Liang XQ, Cao EH, Zhang $Y$ and Qin JF (2003) P53-induced gene 11 (PIG11) involved in arsenic trioxide-induced apoptosis in human gastric cancer MGC-803 cells. Oncol Rep 10, 1265-1269

19. Higgins DF, Kimura K, Bernhardt WM et al (2007) Hypoxia promotes fibrogenesis in vivo via HIF-1 stimulation of epithelial-to-mesenchymal transition. J Clin Invest 117, 3810-3820

20. Schofield CJ and Ratcliffe PJ (2004) Oxygen sensing by HIF hydroxylases. Nat Rev Mol Cell Biol 5, 343-354

21. Noman MZ, Buart S, Romero P et al (2012) Hypoxiainducible miR-210 regulates the susceptibility of tumor cells to lysis by cytotoxic T cells. Cancer Res 72, 46294641

22. Huang X, Ding L, Bennewith KL et al (2009) Hypoxiainducible mir-210 regulates normoxic gene expression involved in tumor initiation. Mol Cell 35, 856-867 